\title{
Experimental infections of mosquitoes with severe fever with thrombocytopenia syndrome virus
}

\author{
Shu-Yi Liang ${ }^{1 \dagger}$, Hong-Liang Chu ${ }^{1 \dagger}$, Xi-Ling Guo ${ }^{1 \dagger}$, Wei Wang ${ }^{2}$, Hong-Na Chen ${ }^{1}$, Yu-Fu Zhang ${ }^{1}$, Yin Chen ${ }^{1}$, Tao Wu', \\ Chang-Jun Bao ${ }^{1}$ and Ming-Hao Zhou ${ }^{1 *}$
}

\begin{abstract}
Background: Severe fever with thrombocytopenia syndrome (SFTS) is a newly identified emerging infectious disease, which is caused by a novel bunyavirus (termed SFTSV) in Asia. Although mosquitoes have not been identified as the primary vectors, as revealed by epidemiological surveys, their role in transmitting this SFTSV as a suspicious vector has not been validated.

Findings: In this study, we conducted experimental infections of mosquitoes with SFTSV to examine the role of mosquitoes in the transmission of the virus. We did not detect viral replication in Culex pipiens pallens, Aedes aegyptis and Anopheles sinensis as revealed by qRT-PCR assay. In addition, we failed to isolate SFTSV from the Vero cells cultured with suspensions of SFTSV-infected mosquitoes.

Conclusion: The results of the present study demonstrate little possibility that mosquitoes act as vectors for the emerging pathogen SFTSV.

Keywords: Severe fever with thrombocytopenia syndrome virus (SFTSV), Vector, Culex pipiens pallens, Aedes aegyptis, Anopheles sinensis, Real-time RT-PCR
\end{abstract}

\section{Multilingual abstracts}

Please see Additional file 1 for translations of the abstract into six official working languages of the United Nations.

\section{Background}

Since 2009, sporadic cases with clinical manifestations of acute onset of fever, low white blood cell and platelet counts, high levels of alanine and aspartate transaminases, and proteinuria, have been found in China [1]. A novel bunyavirus, which was then termed severe fever with thrombocytopenia syndrome virus (SFTSV) in 2011, was found to be associated with this disorder [2, 3]. Moreover, confirmed SFTS cases have been reported from Japan and South Korea in recent years [4, 5]. Severe fever with thrombocytopenia syndrome (SFTS), an

\footnotetext{
* Correspondence: zmh@jscdc.cn

${ }^{\dagger}$ Equal contributors

${ }^{1}$ Jiangsu Provincial Center for Disease Control and Prevention, No. 172

Jiangsu Road, Nanjing, People's Republic of China

Full list of author information is available at the end of the article
}

emerging infectious disease with mortality up to more than $10 \%$, has become an increasingly important public health concern in China [6].

As a novel virus identified in the genus Phlebovirus of the family Bunyaviridae, little is known about the natural transmission cycle. Haemaphysalis longicornis, a widely distributed tick species, has been supposed to act as a vector of SFTSV [7]. However, most patients denied history of tick bite before their onset of SFTS [8]. In addition, epidemiological analyses of a cluster of cases infected with SFTSV showed evidence of person-toperson transmission of the virus through direct blood contact with the patients [9-12]. Nevertheless, there is little knowledge on the vector during the person-toperson transmission cycle of SFTSV. Mosquitoes are probably the most common vectors of infectious diseases [13], and they are identified as major vectors for transmission of multiple Bunyaviridae viruses [14]. The present study aimed to investigate the role of mosquitoes in the transmission of SFTSV. 


\section{Methods}

This study was approved by the Ethical Review Committee of Jiangsu Provincial Center for Disease Prevention and Control (JSCDC). All animal experiments were performed in strict accordance with the Guidelines for Laboratory Animal Care and Management of China [15]. The SFTSV strain JS-2010-014 used in this study was initially isolated from a patient in 2010 in a bio-safety level-2 (BSL-2) facility of Jiangsu Province.

Experimental infection was performed in a BSL-2 laboratory in JSCDC. After starvation for $12 \mathrm{~h}$, three dominant mosquito species Culex pipiens pallens, Aedes aegypti and Anopheles sinensis were fed with sugar water, SFTSV strain JS-2010-014, and blood of guinea pigs (Nanjing Anlimo Technology Co., Ltd.; Nanjing, China) at a ratio of $1: 1: 6$ (blood mixture sample) for $4 \mathrm{~h}$. Then, each species was randomized to 5 cages, of 100 to 150 mosquitoes in each cage, and was fed with sugar water for $4,24,48,72$, and 96 h, respectively, while uninfected mosquitoes served as controls. At assigned time points, 20 female mosquitoes of each species were collected, washed three times with physiological saline and ground with maintenance medium to yield mosquito suspensions.

SFTSV RNA was isolated from mosquito suspensions, and viral loads were determined by using TaqMan quantitative real-time PCR (qRT-PCR) assay as described previously [16]. PCR cycle threshold (Ct) values of 35 or less were considered positive, while $\mathrm{Ct}$ value of $>35$ was defined negative. All samples were subjected to SFTSV RNA detection as described above.

Once qRT-PCR showed positive, the samples were used for virus isolation. SFTSV-positive mosquito suspensions were centrifuged, and the supernatant was seeded onto single-layer African green monkey kidney Vero cells. Cytopathic effect of Vero cells was assessed using indirect immunofluorescence assay (IFA). Vero cells were grown on an 8-well Millicell EZ slide (Millipore; Billerica, MA, USA) at $37{ }^{\circ} \mathrm{C}$ for $36 \mathrm{~h}$, and cells were fixed by treatment with acetone for at $20{ }^{\circ} \mathrm{C} 10 \mathrm{~min}$. Mouse anti-SFTSV NP polyclonal antisera were incubated with the fixed cells at $37{ }^{\circ} \mathrm{C}$ for $30 \mathrm{~min}$. Bound antibodies were detected using fluoresce in isothiocyanate (FITC)-conjugated anti-mouse antibodies (KPL; Gaithersburg, MD, USA) that were diluted by PBS containing 0.01\% Evens blue, and observed under an Eclipse TS100 inverted fluorescence microscope (Nikon; Kanagawa, Japan). Cell passage was performed blindly for three passages. Loads of SFTSV at each passage were assessed using qRT-PCR assay. Normal Vero cells without inoculation served as a blank control, and SFTSV-infected Vero cells were used as a positive control.

\section{Findings}

qRT-PCR assay revealed positive SFTSV in $C x$. pipiens pallens that was fed with sugar water for $4,24,48$, and
$72 \mathrm{~h}$, Ae. Aegypti given sugar water for 4, 24, and $48 \mathrm{~h}$, and $A n$. Sinensis fed with sugar water for 4, 24, 48, 72 and $96 \mathrm{~h}$ (Table 1). An increasing tendency of $\mathrm{Ct}$ values was observed in all three mosquito species with the extension of feeding (Fig. 1), indicating no viral replication detected in mosquitoes. Following inoculation of the supernatant of SFTSV-positive mosquito suspensions, Vero cells developed cytopathic effect in all three passages of $C x$. pipiens pallens that was fed with blood mixture samples for $4 \mathrm{~h}$, and qRT-PCR detected positive SFTSV. However no cytopathic effect was seen in Vero cells seeded with other two Ae. Aegypti or An. Sinensis suspension supernatant, and negative SFTSV was detected by qRT-PCR assay (Fig. 2a-e).

Identification of a vector requires an evidence of isolation of pathogen and evidence of vector transmission capability and ecology [17]. In this study, artificial infection of mosquitoes with SFTSV was performed to assess the capability to transmit the virus. qRT-PCR assay showed that three mosquito species Cx. Pipiens pallens, Ae. Aegyptis and An. sinensis were successfully infected with artificial blood mixture sample containing SFTSV, and viral loads gradually declined with the extension of breeding time after mosquitoes were fed with SFTSV. In addition, no viral replication was detected However, SFTSV was isolated from Vero cells inoculated with SFTSV-positive Cx. Pipiens pallens suspensions, while the virus was not isolated from cells seeded with suspension supernatant of Ae. Aegyptis and An. sinensis. The results of the current study demonstrate that the viral ability of SFTSV almost disappears in mosquitoes, and no viral replication is detected after 4 to $24 \mathrm{~h}$ of virus feeding, which proves that mosquitoes have no ability to transmit SFTSV. Isolation of SFTSV from Vero cells inoculated with SFTSV-positive Cx. Pipiens pallens suspensions after 4-h virus feeding may be caused by incomplete wash of SFTSV, which is mechanically carried on the body surface of Cx. Pipiens pallens.

\section{Conclusions}

Our findings demonstrate that mosquitoes are not vectors for transmission of SFTSV. Currently, ticks are widely accepted as vectors of SFTSV [1, 2, 18-20].

Table 1 qRT-PCR assay detects viral loads of SFTSV in Culex pipines pallens, Aedes aegyptis and Anopheles sinensis following different breeding time

\begin{tabular}{llll}
\hline $\begin{array}{l}\text { Breeding time } \\
\text { (h) }\end{array}$ & Ct value & & \\
\cline { 2 - 4 } & Culex pipiens pallens & Aedes aegyptis & Anopheles sinensis \\
\hline 4 & Positive (24.59) & Positive (25.58) & Positive (24.84) \\
24 & Positive (24.15) & Positive (28.82) & Positive (28.69) \\
48 & Positive (27.89) & Positive (30.03) & Positive (31.06) \\
72 & Positive (28.49) & Negative (>35) & Positive (30.61) \\
96 & Negative $(>35)$ & Negative (>35) & Positive (33.94) \\
\hline
\end{tabular}




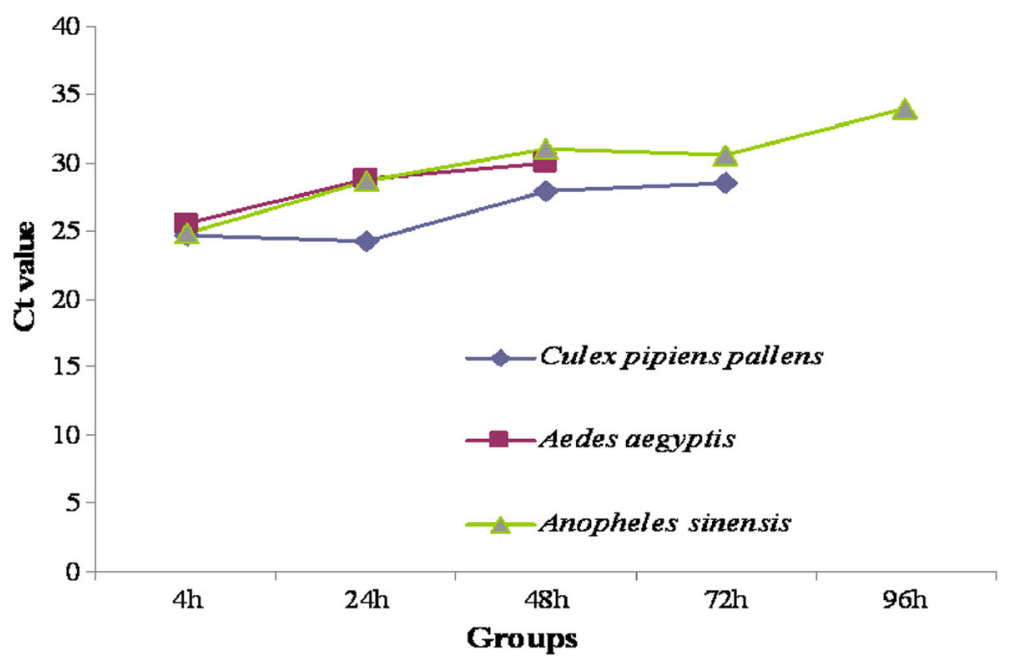

Fig. 1 RT-PCR assay detects viral loads of SFTSV in Cx. Pipiens allens, Ae. Egypti nd An. inensis at different breeding time following feeding blood mixture samples

However, further studies are needed to validate previous hypothesis. Since SFTS caused by SFTSV has a high fatality, and is an increasingly public health concern, health education pertaining to knowledge on correct, effective prevention of vector control should be strengthened. Notably, individual protection targeting high-risk population is of great significance to guide the prevention of SFTS. In addition, health sections require intensification of monitoring and clinical therapy of this emerging infectious disease and the ability of early identification and early diagnosis should be improved to reduce the incidence of SFTS and protect human health.



Fig. 2 ytopathic effect of Vero cells. a Cytopathic effect observed in Vero cells infected with SFTSV JS-2010-014 strain; b Cytopathic effect observed in Vero cells inoculated with blood mixture sample; c Cytopathic effect seen in normal Vero cells; d Cytopathic effect found in Vero cells inoculated with Cx. Pipiens allens uspensions following $24 \mathrm{~h}$ feeding of blood mixture samples; e Cytopathic effect found in Vero cells inoculated with $x$. pipiens allens uspensions following $4 \mathrm{~h}$ feeding of blood mixture samples 


\section{Additional file}

Additional file 1: Multilingual abstracts in the six official working languages of the United Nations. (PDF $785 \mathrm{~kb}$ )

\section{Abbreviations}

PCR: Polymerase chain reaction; SFTS: Severe fever with thrombocytopenia syndrome; SFTSV: Severe fever with thrombocytopenia syndromevirus

\section{Acknowledgements}

We would like to thank all study participants who were involved and contributed to the data collection. We thank editors and reviewers for their warm works and valuable comments.

\section{Funding}

This work was supported by: the National Natural Science Foundation of China (81373055), Science \& Technology Demonstration Project for Emerging Infectious Diseases Control and Prevention(BE2015714)and The key discipline of epidemic(ZDXKA2016008).

\section{Availability of data and materials}

Data sharing not applicable to this article as no datasets were generated or analyzed during the current study.

\section{Authors' contributions}

$X L G$ and HLC conceived and designed the study. SYL and XLG collected the data. XLG, SYL, WW, HNC, YFZ, YC, TW, CJB implemented the study. SYL prepared the manuscript. $\mathrm{MHZ}$ revised and finalized the manuscript. All of the authors read and approved the final version of the manuscript.

\section{Competing interests}

The authors declare that they have no competing interests.

\section{Consent for publication}

Not applicable.

\section{Ethics approval and consent to participate}

This study was approved by the Ethics Review Committee of Jiangsu Province, Jiangsu Provincial Center for Disease Control and Prevention.

\section{Author details}

${ }^{1}$ Jiangsu Provincial Center for Disease Control and Prevention, No. 172 Jiangsu Road, Nanjing, People's Republic of China. ${ }^{2}$ Jiangsu Institute of Parasitic Diseases, No. 117 Yangxiang, Meiyuan, Wuxi, People's Republic of China

Received: 28 November 2016 Accepted: 9 March 2017

Published online: 01 June 2017

\section{References}

1. Li DX. A highly pathogenic new bunyavirus emerged in China. Emerg Microbes Infect. 2013;1:e1.

2. Yu XJ, Liang MF, Zhang SY, Liu Y, Li JD, Sun YL. Fever with thrombocytopenia associated with a novel bunyavirus in China. N Engl J Med. 2011;364:1523-32.

3. Xu BL, Liu LC, Huang XY, Ma H, Zhang Y, Du Y. Metagenomic analysis of fever, thrombocytopenia and leukopenia syndrome (FTLS) in Henan Province, China: discovery of a new bunyavirus. PLoS Pathog. 2011;7:e1002369.

4. Takahashi T, Maeda K, Suzuki T, Ishido A, Shigeoka T, Tominaga T, et al. The first identification and retrospective study of severe fever with thrombocytopenia syndrome in Japan. J Infect Dis. 2014;209:816-27.

5. Kim KH, Yi J, Kim G, Choi SJ, Jun Kl, Kim NH, et al. Severe fever with thrombocytopenia syndrome, South Korea, 2012. Emerg Infect Dis. 2013:19:1892-4

6. Liu Q, He B, Huang SY, Wei F, Zhu XQ. Severe fever with thrombocytopenia syndrome, an emerging tick-borne zoonosis. Lancet Infect Dis. 2014;14:763-72.
7. Liu H, Li Z, Wang Z, He B, Wang S, Wei F, Tu C, Liu Q, et al. The first molecular evidence of severe fever with thrombocytopenia syndrome virus in ticks in Jinlin, Northeastern, China. Ticks Tick Borne Dis. 2016;7:1280-3.

8. Liu S, Chai C, Wang C, Amer S, Lv H, He H. Systematic review of severe fever with thrombocytopenia syndrome: virology, epidemiology, and clinical characteristics. Rev Med Virol. 2014;24:90-102

9. Gai Z, Liang M, Zhang Y, Zhang S, Jin C, Wang SW. A family cluster of infections by a newly recognized bunyavirus in eastern China, 2007: further evidence of person-to-person transmission. Clin Infect Dis. 2011;53:1208-14.

10. Bao CJ, Qi X, Wang H. A novel bunyavirus in China. N Engl J Med. 2011;365:862-3.

11. Bao CJ, Guo XL, Qi X, Hu JL, Zhou MH, Varma JK. A family cluster of infections by a newly recognized bunyavirus in eastern China, 2007: further evidence of person-to-person transmission. Clin Infect Dis. 2011;53:1208-14.

12. Liu Y, Li Q, Hu W, Wu J, Wang Y, Mei L. Person-to-person transmission of severe fever with thrombocytopenia syndrome virus. Vector Borne Zoonotic Dis. 2012;12:156-60.

13. Tolle MA. Mosquito-borne diseases. Curr Probl Pediatr Adolesc Health Care. 2009:39:97-140.

14. Beaty BJ, Bishop DH. Bunyavirus-vector interactions. Viruses. 2014;6:4373-97.

15. State Council of China. Experimental Animal Management Regulation of People's Republic of China. 2011

16. Li Z, Qi X, Zhou M, Bao C, Hu J, Wu B. A two-tube multiplex real-time RTPCR assay for the detection of four hemorrhagic fever viruses: severe fever with thrombocytopenia syndrome virus, Hantaan virus, Seoul virus, and denque virus. Arch Virol. 2013;158:1857-63.

17. Kilpatrick AM, Randolph SE. Drivers, dynamics, and control of emerging vector-borne zoonotic diseases. Lancet. 2014:380:1946-55.

18. Weaver SC, Brault AC, Kang W, Holland JJ. Genetic and fitness changes accompanying adaptation of an arbovirus to vertebrate and invertebrate cells. J Virol. 1999:73:4316-26.

19. Weaver SC, Barrett AD. Transmission cycles, host range, evolution and emergence of arboviral disease. Nat Rev Microbiol. 2004:2:789-801.

20. Powers AM, Roehrig JT. Alphaviruses. Methods Mol Biol. 2011;665:17-38.
Submit your next manuscript to BioMed Central and we will help you at every step:

- We accept pre-submission inquiries

- Our selector tool helps you to find the most relevant journal

- We provide round the clock customer support

- Convenient online submission

- Thorough peer review

- Inclusion in PubMed and all major indexing services

- Maximum visibility for your research

Submit your manuscript at www.biomedcentral.com/submit 